\title{
EMBEDDING PARTIALLY ORDERED SPACES IN TOPOLOGICAL SEMILATTICES ${ }^{1}$
}

\author{
LLOYD D. TUCKER
}

\begin{abstract}
A partial order $\Gamma$ on a compact space $S$ is called continuous if $\Gamma$ is a closed subset of $S \times S$. In this paper, we define and study an embedding $\Phi$ of the arbitrary compact continuously partially ordered space $(S, \Gamma)$ into a corresponding compact topological semilattice $S_{\Gamma}$. We show that the structure of $S_{\Gamma}$ entirely determines the structure of $(S, \Gamma)$. We prove that the inverse images under $\Phi$ of components in $S_{\Gamma}$ are the order components of $(S, \Gamma)$, where elements $a$ and $b$ of $S$ are defined to be in the same order component of $(S, \Gamma)$ if there exists no continuous monotonic map $f:(S, \Gamma) \rightarrow\{0,1\}$ which separates $a$ and $b$. Finally, we show that $S_{\Gamma}$ is connected if and only if $(S, \Gamma)$ has only one order component.
\end{abstract}

Notation. If $S$ is a topological space, we denote the space of nonempty closed subsets of $S$ by $2^{S}$. If $\left\{V_{j} \mid j=1, \cdots, n\right\}$ is a set of open subsets of $S$, we let $\left\langle V_{1}, \cdots, V_{n}\right\rangle=\left\{X \subset 2^{S} \mid X \cap V_{j} \neq \varnothing\right.$ for each $j \leqq n$ and $X \subset V_{1} \cup$. $\left.\cdots \cup V_{n}\right\}$. We give $2^{S}$ the finite topology [1], a basis for which is $\left\{\left\langle V_{1}, \cdots, V_{n}\right\rangle \mid V_{1}, \cdots, V_{n}\right.$ are open subsets of $\left.S\right\}$.

A quasi order is a transitive reflexive binary relation. A partial order is an antisymmetric quasi order. If $\Gamma$ is a quasi order on the Hausdorff space $S$ and $\Gamma$ is closed with respect to the product topology on $S \times S$, then $\Gamma$ is a continuous quasi order. We write CCQOTS (CCPOTS) for "compact continuously quasi (partially) ordered topological space(s)." Let $(S, \Gamma)$ and $(T, \Omega)$ be CCQOTS. If $x \in S$ and $A \subset S$, then we let $x \Gamma=\{y \in S \mid(x, y) \in \Gamma\}$, and $A \Gamma=\bigcup\{a \Gamma \mid a \in A\}$. It is easy to see [4] that if $A$ is compact then $A \Gamma$ is closed. We say $f:(S, \Gamma) \rightarrow(T, \Omega)$ is monotonic if $\hat{f}: S \rightarrow T$ is a function such that if $(a, b) \in \Gamma$, then $(f(a), f(b)) \in \Gamma^{\prime}$. If $f:(S, \Gamma) \rightarrow(T, \Omega)$ is monotonic, one-to-one, and onto, and $f^{-1}:(T, \Omega) \rightarrow(S, \Gamma)$ is monotonic, then $f$ is an isomorphism. We let

$$
\begin{aligned}
E(\Gamma) & =\{(x, y) \in S \times S \mid(x, y),(y, x) \in \Gamma\}, \\
\Gamma / E(\Gamma) & =\{(a, b) \in S / E(\Gamma) \times S / E(\Gamma) \mid \text { if } x \in a \text { and } y \in b \text { then }(x, y) \in \Gamma\},
\end{aligned}
$$

Received by the editors April 30, 1971.

AMS 1970 subject classifications. Primary 54F05; Secondary 06A20.

Key words and phrases. Continuous orders, embedding, topological semilattice, order component, order connected.

1 This research was supported in part by a grant from the National Science Foundation.

(c) American Mathematical Society 1972 


$$
\begin{aligned}
U(\Gamma) & =\left\{(A, B) \in 2^{S} \times 2^{S} \mid A \Gamma \supset B \Gamma\right\}, \\
S^{\prime} & =2^{S} / E(U(\Gamma)) \text { and } \Gamma^{\prime}=U(\Gamma) / E(U(\Gamma)) .
\end{aligned}
$$

We say $(T, *)$ is a topological semilattice if $*$ is a continuous commutative associative operation on the space $T$ such that $t * t=t$ for each $t \in T$.

Proposition 1. If $(S, \Gamma)$ is a CCPOTS, then $\left(2^{S}, U(\Gamma)\right)$ is a CCQOTS, and $\left(S^{\prime}, \Gamma^{\prime}\right)$ is a CCPOTS.

Proof. Well known.

Definition of $S_{\Gamma}$. If $\left(S, I^{\prime}\right)$ is a CCPOTS, let $F(\Gamma)$ be the set of all continuous monotonic $f:(S, \Gamma) \rightarrow I$, where $I$ is the unit interval with the usual topology and order. Let $C=C(F(\Gamma))$ be the cube $\{g: F(\Gamma) \rightarrow I\}$, with the product topology and the natural partial order $\leqq$, defined by pointwise minimum. Specifically, we define $*: 2^{C} \rightarrow C$ by $\pi_{f}(*(A))=\inf \left\{\pi_{f}(a) \mid a \in A\right\}$ where $\pi_{f}$ is the projection map, and we say $a \leqq b$ if $*(\{a, b\})=a$. Let $\Phi: S \rightarrow C$ be the usual embedding of $S$ into $C$ defined by $\pi_{f}(\Phi(s))=f(s)$. We let $S_{\mathrm{r}}$, be the smallest (topologically) closed subsemilattice of $C$ which contains $\Phi(S)$.

Note. If $(S, \Gamma)$ is a CCPOTS, then by [2, p. 30], $F(\Gamma)$ separates points of $S$, so $\Phi$ is one-to-one. Thus $(D$ is a homeomorphism into $C$. Also, the partial order $\Gamma$ agrees with $\leqq$ in the sense that $(a, b) \in \Gamma$ if and only if $\Phi(a) \leqq \Phi(b)$. Thus, we assume without loss of generality that $S$ is a subspace of $C$.

Proposition 2. If $(S, \Gamma)$ is a CCPOTS, and $i:\left(2^{S}, U(\Gamma)\right) \rightarrow S$ is defined by $i(A)=*(A)$. then $i$ is a continuous monotonic extension of the embedding $\dot{\Phi}:(S, \Gamma) \rightarrow S_{\Gamma}$ such that $i$ is onto $S_{\Gamma}$, and if $i(A) \leqq i(B)$ for some $A, B \in 2^{S}$, then $(A, B) \in U(\Gamma)$.

Proof. Let $T=\left\{*(A) \mid A \in 2^{S}\right\}$. Since $*$ is a continuous operation and $2^{S}$ is compact, $T$ is closed. Clearly, $T$ is a subsemilattice of $C$ containing $S$, so $S_{\Gamma} \subset T$. Let $\mathscr{F}(S)$ be the set of all nonempty finite subsets of $S$. By $\left[1\right.$, p. 156], $\mathscr{F}(S)$ is dense in $2^{S}$. Since $*(A) \in S_{\Gamma}$ for each $A \in \mathscr{F}(S)$, we see that $T \subset S_{\Gamma}$. Therefore $T=S_{\Gamma}$. One may now show $i$ satisfies the desired conclusion by applying the theorem of Nachbin [2, p. 30] which states that if $a \in S, B \in 2^{S}$, and $a \notin B \Gamma$, then there exists $f \in F(\Gamma)$ such that $f$ separates $a$ and $B$.

COROLLARY 3. If $(S, \Gamma)$ is a CCPOTS and $q: 2^{S} \rightarrow S^{\prime}$ is the quotient map, then there exists an isomorphism $j:\left(S^{\prime}, \Gamma^{\prime}\right) \rightarrow S_{\Gamma}$ such that $j$ is a homeomorphism and the following diagram commutes.

Proof. Straightforward. 


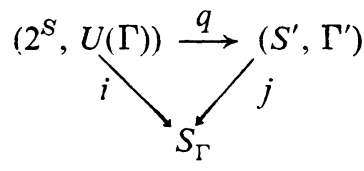

Definition. If $X$ is a topological semilattice and $A \subset X$, then $A$ is a minimal closed generating subset of $X$ if $A$ is closed, $A$ generates $X$ in the sense that the only closed subsemilattice of $X$ containing $A$ is $X$, and no closed proper subset of $A$ generates $X$.

Proposition 4. If $(S, \Gamma)$ is a CCPOTS, then $\Phi(S)$ is the unique minimal closed generating subset of $S_{\Gamma}$.

PrGor. Suppose on the contrary that there exists a closed generating subset $A$ of $S_{\Gamma}$ such that $S$ is not a subset of $A$. Let $T=\left\{*(D) \mid D \in 2^{A}\right\}$. One can show $T=S_{\Gamma}$ by using an argument similar to the proof of Proposition 2. Let $s$ be an element of $S \backslash A=\{x \in S \mid x \notin A\}$.

There exists $B \in 2^{A}$ such that $*(B)=s$. Let $P=\left\{E \in 2^{i s} \mid i(E) \in B\right\}$. Since $P$ is closed in $2^{S}$, one can show $\bigcup P$ is closed in $S$. We want to show $\bigcup P \subset s \Gamma$. Suppose on the contrary there exists $x \in \bigcup P \mid s \Gamma$. Then by [2, p. 30] there exists $f \in F(\Gamma)$ such that $f(x)=0$ and $f(t)=1$ for each $t \in s \Gamma$. Clearly, $\pi_{f}(i(\bigcup P))=0$ and $\pi_{f}(s)=1$. But $i(\bigcup P)=*(\bigcup P)=*(B)=s$. This contradiction indicates that $\bigcup P \subset s \Gamma$. Similarly, one can show $s \in \bigcup P$. Thus, there exists $E \in P$ such that $s \in E \subset s \Gamma$. Since $(E,\{s\}),(\{s\}, E) \in U(\Gamma)$ and $i$ is monotonic, we see that $i(E)=i(\{s\})=s$. Thus $s \in B \subset A$. This contradiction indicates $S \subset A$. Therefore, $S$ is a minimal generating closed subset of $S_{\Gamma}$.

'Corollar'y' 5. If $(S, \Gamma)$ and $(T, \Omega)$ are CCPOTS, and $S_{\Gamma}$ and $T_{\Omega}$ are isomorphic and homeomorphic, then $(S, \Gamma)$ and $(T, \Omega)$ are isomorphic and homeomorplic.

Proof. By Proposition 4, we can recover the topological and order structures of $(S, \Gamma)$ and $(T, \Omega)$ from the topological and order structures of $S_{\Gamma}$ and $T_{\Omega}$ respectively.

DEFINITION. In [3], the author defined the relation $C_{3}(\Gamma)$ on the CCPOTS $(S, \Gamma)$ by

$$
\begin{aligned}
& C_{3}(\Gamma)=\{(a, b) \in S \times S \mid \text { there exists no } f \in F(\Gamma) \text { such } \\
& \text { that the image of } f \text { is }\{0,1\} \text { and } f(a) \neq f(b)\} .
\end{aligned}
$$

The equivalence classes of $C_{3}(\Gamma)$ are called order components. If $C_{3}(\Gamma)=$ $S \times S$, then $(S, \Gamma)$ is order connected.

Proposition 6. If $(S, \Gamma)$ is a CCPOTS and $a, b \in S$, then $a$ and $b$ lie in the same order component of $(S, \Gamma)$ if and only if $\Phi(a)$ and $\Phi(b)$ lie in the same component of $S_{i}$. 
Proof. First, suppose $(a, b) \in S \times S \backslash C_{3}(\Gamma)$. There exists $f \in F(\Gamma)$ such that the image of $f$ is $\{0,1\}$, and $f(a) \neq f(b)$. Thus, $S_{\Gamma}=\pi_{f}^{-1}(\{0,1\})$, and $a$ and $b$ lie in the disjoint open sets $\pi_{f}^{-1}(0)$ and $\pi_{f}^{-1}(1)$ respectively, so $\Phi(a)=a$ and $\Phi(b)=b$ lie in distinct components of $S$.

Now suppose $\Phi(a)$ and $\Phi(b)$ lie in distinct components of $S_{\Gamma}$. By [3], the order components and the components of a compact topological semilattice are identical, so there exists $g \in F(\leqq)$ such that the image of $g$ is $\{0,1\}$ and $g(a) \neq g(b)$. Clearly, $g \circ \Phi \in F(\Gamma)$, the image of $g \circ \Phi$ is $\{0,1\}$. and $g \circ \Phi(a) \neq g_{\circ} \Phi(b)$. Thus, $a$ and $b$ lie in distinct order components of $(S, \Gamma)$

Proposition 7. If $(S, \Gamma)$ is a CCPOTS, then $S$ is order connected if and only if $S_{\Gamma}$ is connected.

Proof. $\quad(\Leftrightarrow)$ Apply Proposition 6.

$\Leftrightarrow$ Suppose $S_{\Gamma}$ is not connected. By [3], the order components and the components of $S_{\Gamma}$ are identical, so there exists $g \in F(\leqq)$ such that the image of $g$ is $\{0,1\}$. Let $\mathscr{F}(S)$ be the set of finite subsets of $S$. Since $(g \circ i)^{-1}(0)$ is a nonempty open subset of $2^{S}$, and since by $[1] \mathscr{F}(S)$ is dense in $2^{S}$, there exists $A \in \mathscr{F}(S) \cap(g \circ i)^{-1}(0)$. Let $B \in(g \circ i)^{-1}(1)$, and let $b \in B$. Define $F(U(\Gamma))$ just as in the case where the order is a partial order. Clearly $g \circ i \in F(U(\Gamma))$, $g \circ i(\{b\})=1$, and $g \circ i(A \cup\{b\})=0$. Let $D$ be a subset of $A \cup\{b\}$ such that $b \in D \in(g \circ i)^{-1}(0)$, and no proper subset of $D$ which contains $b$ is an element of $(g \circ i)^{-1}(0)$. There exists $d \in D \backslash\{b\}$.

Define $h: S \rightarrow 2^{S}$ by $h(s)=\{s\} \cup(D \backslash\{d\})$. Clearly, $h:(S, \Gamma) \rightarrow\left(2^{S}, U(\Gamma)\right)$ is continuous and monotonic, so $g \circ i \circ h \in F(\Gamma)$. But $g \circ i \circ h(d)=0, g \circ i \circ h(b)=1$, and the image of $g \circ i \circ h$ is $\{0,1\}$. Thus $(S, \Gamma)$ is not order connected.

\section{REFERENCES}

1. E. A. Michael, Topologies on spaces of subsets, Trans. Amer. Math. Soc. 71 (1951), 152-182. MR 13, 54.

2. L. Nachbin, Sur les espaces topologiques ordonnés, C. R. Acad. Sci. Paris 226 (1948), 381-382: English transl. in Topology and order, Van Nostrand Math. Studies, Van Nostrand, Princeton, N.J., 1965. MR 9, 367; MR 36 \#2125.

3. Lloyd D. Tucker, Generalized components and continuous orders (to appear).

4. L. E. Ward, Jr., Concerning Koch's theorem on the existence of arcs, Pacific J. Math. 15 (1965), 347-355. MR 31 \#6206.

Department of Mathematics, Boise State College, Boise, Idaho 83707 